\title{
Steinmeere und ihre Strukturen im Saarland
}

\author{
Von Georg SElzer, Saarbrücken
}

Mit 6 Abbildungen im Text

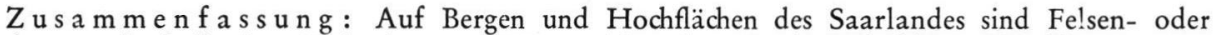
Blockmeere oberhalb einer Höhenlage von $330 \mathrm{~m}$ nicht selten. In den erhaltenen Einzelformen vom stärkst gespaltenen Fels aus Hartstein, dem Schuttmantel der Bergflanken, in den Blockmeeren selbst und besonders in den "Steinringen" sind Vorzeitformen des Eiszeiralters zu erblicken und - worauf stratigraphische Ergebnisse deuten - Erscheinungen der letzten Eiszeit (Würm).

Die erhaltenen Abtragungsprodukte in den mächtigen Hangschutt- und Blockmeer-Bi'dungen deuten auf die sehr starken Verwitterungsvorgänge und damit Formbildungen der Landschaft im Eiszeitalter einerseits, während andererseits die ungestörte Erhaltung der Kleinformen das Feh'en oder die nur schwache Abtragungswirkung in nacheiszeitlicher Zeit hervorheben läßt, so daß damit große Bereiche talfernerer Gebiete des Saarlandes noch heute ein mehr oder weniger genaues Abbi'd der Landschaftsform des Eiszeitalters darstellen.
\end{abstract}

$\mathrm{R}$ é $s \mathrm{u}$ m é : Les mers de blocs de pierres et les coullées de pierres sont très répandues dans les régions sarroises et voisinantes audessus l'altitude de $330 \mathrm{~m}$; ils contiennent des cercles de pierres dresseés et sont toujours en relations avec ses roches-mères, qui possèdent une désagregation forte mais inactive. Ces formes periglaciaires datent de la période wuermienne selon d'une flore d'Eem dans une couche sous-jacente. La richesse et la bonne conservation de ces formes montrent la grande influence des facteurs periglaciaires dans le development du relief récent.

I.

\section{Vorbemerkung:}

Auf vielen Höhen und Hochflächen des Saarlandes, die verschiedenen Landschaftseinheiten angehören, sind Block- und Steínmeere ausgebildet, deren Einzelformen und stratigraphische Bedingungen den Nachweis ihrer Entstehung während der hocheiszeitlichen Perioden des Pleistozäns führen lassen. Einmal soll ihre Darstellung eine Lücke in der Bestandsaufnahme des periglazialen Formenschatzes in unserem Gebiet schließen helfen, andererseits können die fast unverändert erhaltenen Einzelformen der eiszeitlichen Erscheinungen einen Beitrag zu der Frage der Entstehung und Zeitlichkeit der Landschaftsformen unseres Gebietes leisten.

Bislang waren entsprechende Untersuchungen fast ganz auf das Talgebiet der Saar und einiger Nebentäler beschränkt geblieben. Die ausgezeichneten Untersuchungen von Mathias (1936 und 1956), Fischer (1956) und Zandstra (1954) haben deutlich nachgewiesen, daß die Entstehung des Talgebietes der Saar in das Pleistozän fällt, und z. B. in den Einzelstadien der Talerosion und Terrassenbildung eine enge Abhängigkeit zu den einzelnen Eiszeiten besteht. Die Darstellung der Blockmeere und der damit verbundenen Erscheinungen versucht, die Bedeutung der eiszeitlichen Klimawirkungen auf die talfernen Gebiete des Saarlandes zu erweitern, womit Ansätze zu der Auffassung gemacht werden können, daß die jetzige Landschaftsform ein fast unverändertes Abbild der eiszeitlichen Formgebung darstellt und in vielen Gebieten die nacheiszeitlichen Abtragsbedingungungen fast keine morphologischen Veränderung in Groß- wie in Kleinformen hervorgerufen haben.

II.

1. Das Saarland im Eiszeitalter allgemein

Das Saarland war im Pleistozän außerhalb der Vergletscherungen des Inlandeises und der Gebirge gelegen. Unmittelbar glazigene Bildungen lassen sich im Saarland auch nicht in den höchsten Erhebungen des Hunsrücks feststellen. So hat auch die zuerst von MEHLIS (1886) aufgestellte Deutung von Blockmeeren im Pfälzer Wald als Gletschermoränen der 
Eiszeiten der Erklärung als Periglazialbildung weichen müssen. Bedeutsam ist die Lage zu den Vogesen, die im Eiszeitalter eine eindeutige Vergletscherung besessen haben.

Die Erscheinungen der fossilen Frostspalten (P. KESSLER 1915 u. 1927), Eiskeile (Zandstra 1954, Fischer 1956), Brodelböden und dgl. sind Beweise dafür, daß auch im Saarland während der Eiszeiten des Pleistozäns ein tiefgründiger Dauerfrostboden ausgebildet war, der nach Poser (1947) in der Würm-Eiszeit eine sommerliche Auftautiefe von $\mathrm{rd}$. $1,8 \mathrm{~m}$ besessen haben dürtte.

2. Die Blockmeervorkommen im Saarland

Aus dem Saarland sind Blockmeervorkommen bislang noch nicht bekannt geworden. In seiner umfassenden Darstellung der Vorkommen in den deutschen Mittelgebirgen erwähnt Schotт (1931) die Blockmeere im Buntsandstein des Pfälzer Waldes und ganz allgemein die des Rheinischen Schiefergebirges.

Eine vorläufige Darstellung der wichtigsten Vorkommen im Saarland lautet mit Angabe der Höhenlage und der Herkunft der sie aufbauenden Gesteine:

Vorkommen:
Schwarzbruch bei Orscholz
Kaiserweg
Hunscheid
Schimmelkopf
Hunnenring-Dollberg
Weißfels
Mommerich
Trautzberg
Weißelberg
Lindenhübel
Dirmingen
Holzer Höhe

Höhe ü. NN.

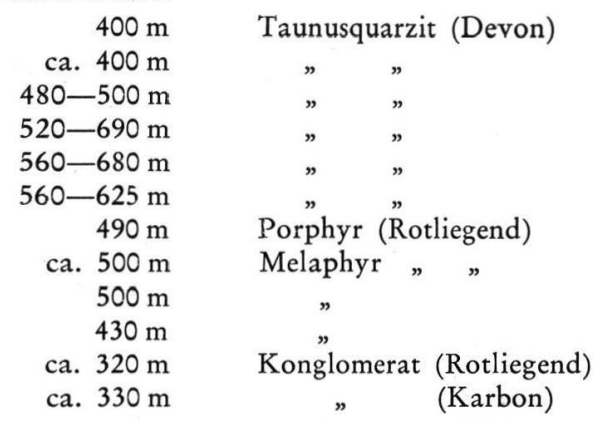

Die weiter nördlich in der Eifel oder im weiteren Hunsrück, dem Pfälzer Bergland (z. B. Donnersberg) und dem Pfälzer Wald (z. B. Kalmit) vorhandenen Vorkommen sind hier nicht speziell aufgenommen worden, zeigen aber eine nennenswerte Häufigkeit und

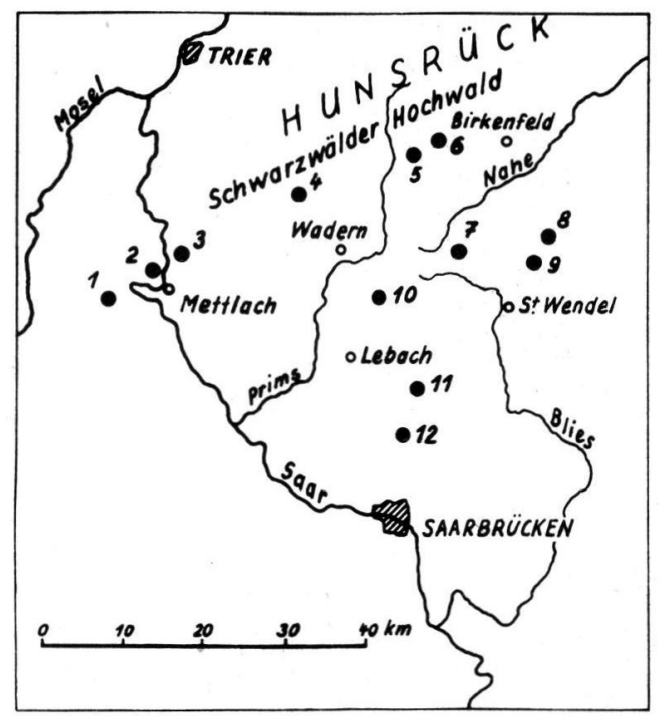

1 Schworzbruch

2 Kaiserweg

3 Hunscheid

4 Schimmelkopf

5 Hunnenring-Dollberg

6 Weißfels

7 Mommerich

8 Troutzberg

9 Weißelberg

10 Lindenhübel

11 Dirmingen

12 Holzer Höhe

Abb. 1. Die Vorkommen von Block- und Steinmeeren im Saarland. 
ähnliche Abhängigkeit wie die des Saarlandes. Ihre Aufzählung läßt aber erkennen, daß die Vorkommen der Blockmeere in den linksrheinischen Mittelgebirgen eine regionale Bedeutung besitzen.

Alle genannten Vorkommen zeigen eine Abhängigkeit von der Höhenlage. Kein Vorkommen liegt unter der Höhe von $320 \mathrm{~m}$. Man gewinnt bei dem Vergleich der verschieden hoch gelegenen Vorkommen den Eindruck, daß die Vorkommen geringerer Höhenlage - unter $400 \mathrm{~m}$ - an Umfang und Mächtigkeit geringer und kleiner sind, als die in größerer Höhenlage. Auch die Vorkommen in der Pfalz sind an Höhen über $400 \mathrm{~m}$ gebunden.

Eine weitere Abhängigkeit der Block- und Felsenmeere ist durch die spezifische Ausbildung der sie zusammensetzenden Gesteine gegeben. Alle Gesteine, die zu einem mehr oder weniger leichten Zerfall durch exogene Einflüsse neigen, fehlen in der Aufstellung ganz, z. B. die Tonschiefer des Altpaläozoikums, die Sandsteine des Karbons und Perms, auch der Buntsandstein der üblichen Ausbildung und ganz die Gesteine des Muschelkalkes, obwohl ihre Vorkommen über $400 \mathrm{~m}$ heraufreichen. Die Muttergesteine der Blockmeere

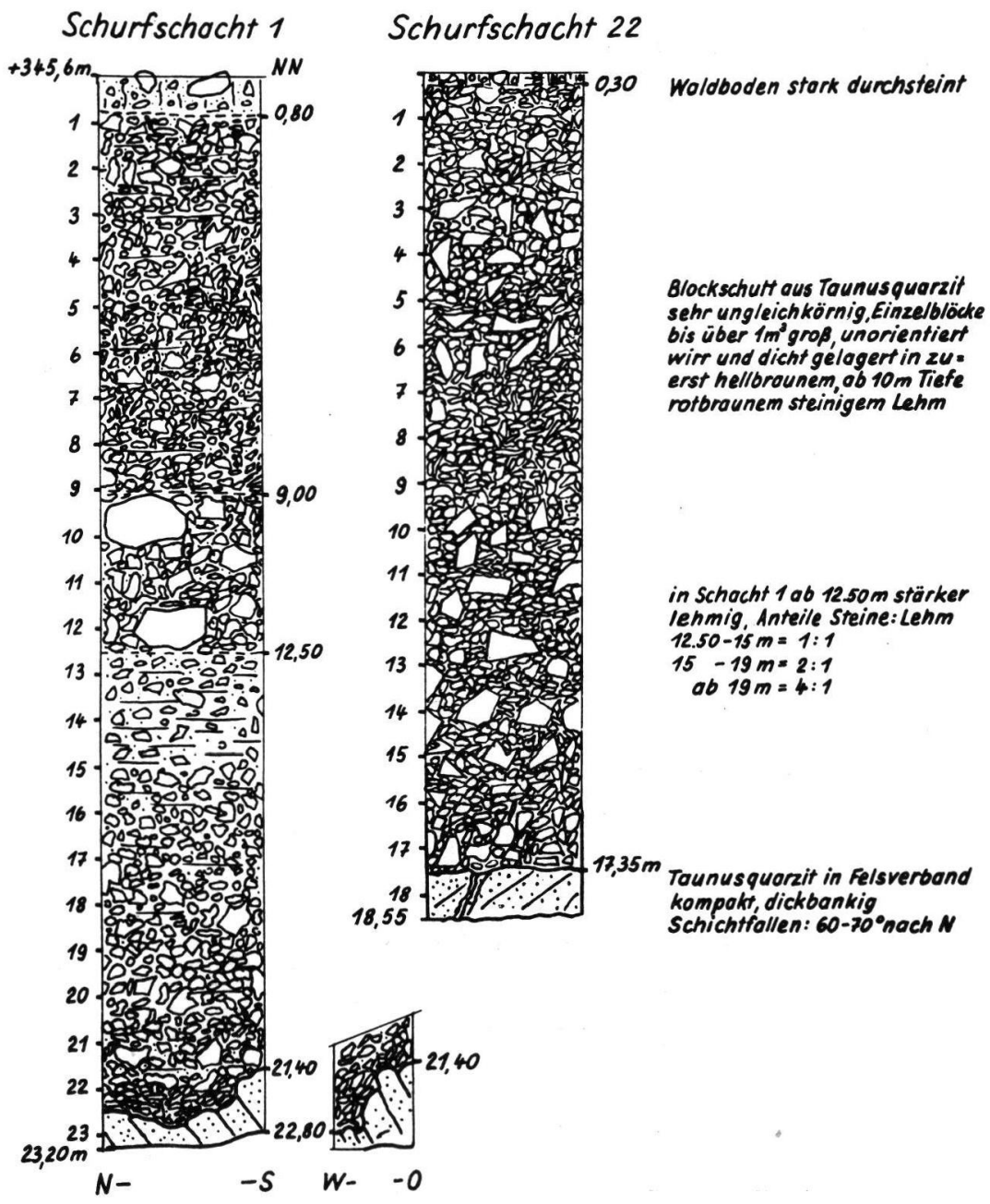

Abb. 2. Profile zweier Schurfschächte im oberen Wellesbachtal b. Orscholz/Saar. 
sind ausschließlich sehr dichte und texturlose, meist monomineralische, aus stabilen Mineralien aufgebaute und stets extrem wetterbeständige Gesteine, also Hartsteine i. e. S.:

Taunusquarzit des Unterdevons,

quarzitische Konglomerate des Karbons und Perms,

feinkörnige Melaphyre und Porphyre des Perms

und nur selten Buntsandstein der quarzitischen Ausbildung.

Die Mächtigkeit und Ausdehnung der Blockmeere ist verschieden. Sie reicht von einer lockeren Überschotterung des sichtbaren Anstehenden bis zu zusammenhängenden Decken von mehr als $1 \mathrm{qkm}$ Fläche. Genau so schwankt die Mächtigkeit von geringen Beträgen auf geneigten Hängen bis zu großen Dicken auf flachem Gelände und Dellen. Selten zeigen natürliche Aufschlüsse das Auflager der Blockvorkommen. In Schurfschächten, die zu Untersuchungen für ein Pumpspeicherprojekt 1952 im oberen Wellesbachtal bei Orscholz (Saarschleife) angelegt worden sind, waren Mächtigkeiten zwischen 15 und $20 \mathrm{~m}$ die Regel und $23 \mathrm{~m}$ die größten, wie Abb. 2 zeigen soll. In der Darstellung des in den Schurfschächten in voller Mächtigkeit aufgeschlossenen Profils der Blockschüttung wurde versucht, die Größe der Einzelbestandteile und die Lagerung maßstäblich und orientiert anzugeben. An vielen, besonders den Vorkommen auf geneigtem Gelände, dürfte mit geringen Dicken und mit einer $10 \mathrm{~m}$ nur selten übersteigenden Dicke der meisten Vorkommen zu rechnen sein.

Die natürlichen Aufschlüsse der Oberfläche wie die in die Tiefe gehenden künstlichen Aufschlüsse lassen Gesteinsstücke von über $1 \mathrm{cbm}$ bis zu Millimetergröße beobachten, wobei sehr auffällig wird, daß die Stücke unter einer Größe (größter Steinquerschnitt) von $1 \mathrm{dm}$ stets scharfkantig, ja, splittrig ausgebildet sind, während alle größeren Formate eine deutliche Abrundung der Kanten besitzen. Einerseits nimmt die Stärke der Kantenrundung mit der Entfernung vom Liefergestein zu. Andererseits konnte in den Schurfschächten eine Abnahme der Kantenrundung in die Tiefe festgestellt werden. Hieraus läßt sich ableiten, daß auf dem Wanderweg der Blöcke Kräfte wirksam waren, die zu einem Abschleifen der Kanten und Verkleinern der Blöcke geführt haben und daß Kräfte und Bewegung in Oberflächennähe stärker waren als in der Tiefe.

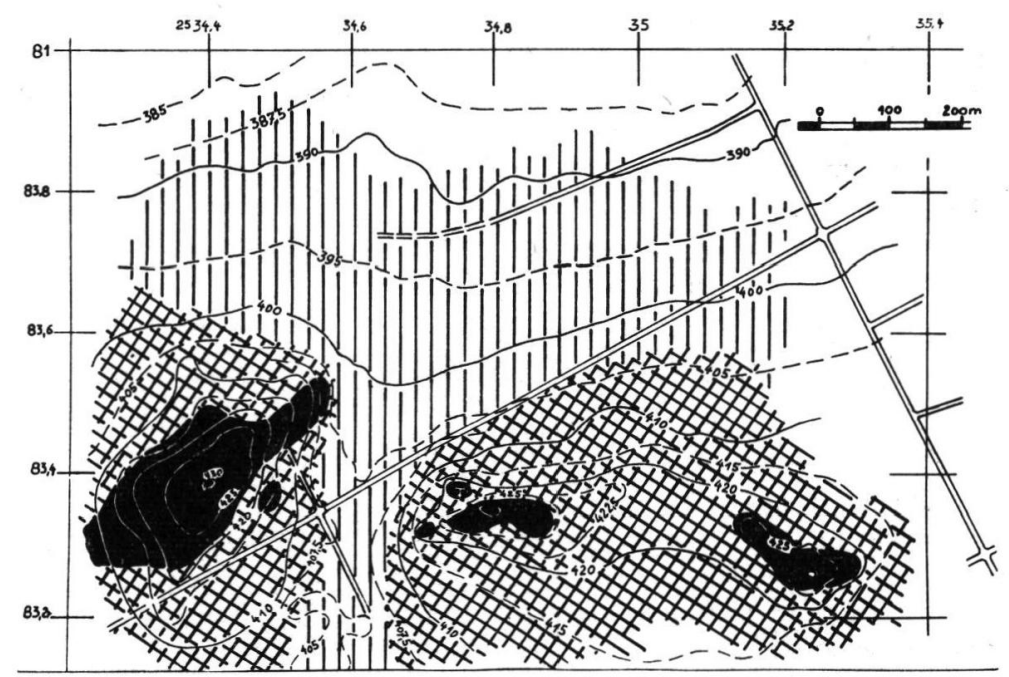

Abb. 3. Das Blockmeer des Schwarzbruches bei Orscholz in seiner Beziehung zum Liefergestein. Schwarz: anstehender Taunusquarzit; kreuzschraffiert: Hangschuttmantel (Kolluvium); Striche: Blockmeer. 
Während in der Tiefe - ab rd. $5 \mathrm{~m}$ - die Einzelblöcke und Gesteinsbruchstücke unorientiert liegen und oft den Eindruck erwecken, als würden sie in einem Lehm isoliert schwimmen, zeigen sie an der Oberfläche eine intensive Verkeilung bis zur Aufspaltung und eine dachziegelartig geregelte Lagerung, die auf eine hangabwärtige Bewegung deutet.

In den meisten Fällen läßt sich nachweisen, daß die Blockströme nicht unmittelbar von dem anstehenden Gebirge gespeist worden sind, sondern von einem beträchtlichen Schuttmantel, der das Anstehende weit verhüllt. So zeigt das Beispiel des Schwarzbruches bei Orscholz ganz instruktive Abhängigkeiten (s. Abb. 3).

Aus einer schwach geneigten Verebnung in rd. $400 \mathrm{~m}$ Höhenlage erheben sich einige Kuppen bis in eine Höhe von $430 \mathrm{~m}$. Nur auf den Kuppenspitzen zeigten sich isolierte Vorkommen aus anstehendem Taunusquarzit, der grob gebankt, sehr kompakt und extrem dicht ausgebildet und mit rd. $12^{\circ}$ nach Süden geneigt ist. Der Quarzit ist von vielen Spalten, vornehmlich senkrecht, durchzogen, die stark - bis einige dm - klaffen und sich nach unten keilförmig verjüngen. Diese Aufspaltung des anstehenden Taunusquarzits entlang derartig klaffender Spalten ist nur an die Oberfläche gebunden, während nahe Steinbrüche in den gleichen Gesteinsbänken keine derartige Aufspaltung zeigen. Andererseits zeigten Bodenbedeckung und Vegetation, daß diese Aufspaltung nicht durch pflanzliche Einwirkung entstanden ist, auch heute nicht weitergebildet wird, so daß in dieser Auflösung des heute wetterbeständigsten Gesteins unserer Gegend eine Vorzeitform zu sehen ist. Diesen Ausbissen des anstehenden Gesteins ist eine sehr breite Zone des Hangschuttes aus eckigen Bruchstücken aller Größen vorgelagert. Diese Hangschuttzone (echtes Kolluvium) hält lokal Neigungen bis $30^{\circ}$ ein und kann nach Aufschlüssen (zur Schottergewinnung) und geoelektrischen Messungen auf weite Strecken bis $20 \mathrm{~m}$ dick werden. Mitunter zeigt der Hangschutt terrassenähnliche Anschwellungen, die hangparallel verlaufen und am gleichen Hang mehrfach übereinander auftreten, wie am Leukerkopf bei Orscholz oder dem Hunnenring.

Meist mit einem deutlichen Hangknick abgesetzt, folgen hangabwärts die eigentlichen Blockströme.

\section{Das Alter der Blockmeere}

In einigen Schurfschächten im oberen Wellesbachtal bei Orscholz (ca. $330 \mathrm{~m}$ ü. NN) ruht die obere und bedeutendere Blockmasse auf einer mehrere Meter dicken Lage eines sehr reinen grauen Tones, dem eine Schicht eines mit vielen organischen Resten durchsetzten, fast schwarzen Tones eingeschaltet ist. Die von Herrn Prof. Dr. Leschik ${ }^{1}$ ) pollenanalytisch untersuchten Proben dieser Schicht ergab eine Zusammensetzung, die nur eine Einstufung in die Eem-Zeit, also in das Riß/Würm-Interglazial, zuläßt. Der diesen Interglazial-Tonen auflagernde Blockschutt mit seinem gänzlich verschiedenen Sedimentationscharakter läßt sich danach zwanglos in die Würm-Eiszeit einordnen.

Wichtig ist ferner, daß der Interglazial-Ton von Orscholz von einer schwächeren Blockschüttung unterlagert wird, die immerhin $5 \mathrm{~m}$ dick wird, wonach mit 2 Zeiten einer Blockmeerbildung zumindest gerechnet werden kann.

\section{Steinringe als Strukturformen der Blockmeere}

Im Blockmeervorkommen des Schwarzbruches bei Orscholz (s. auch Abb. 3), in dem die Hangneigung nur $5^{\circ}$ nach Norden beträgt, sind die ausschließlich aus Taunusquarzit bestehenden großen und kleinsten Komponenten derart angeordnet, daß um einen zentralen Kern aus kleinster Korngröße (bis $1 \mathrm{~cm}$ ) grobes Material ringartig verteilt ist, um das mit größerer Entfernung von der Feinkornmitte immer gröberes Steinmaterial liegt. Die größeren Blöcke in einer Entfernung von rd. $2 \mathrm{~m}$ von der Feinkornmitte zeigen dabei häufig eine Steilstellung in Ringanordnung. Ein solcher eigentümlicher Steinring, der bei

1) Ich bedanke mich an dieser Stelle sehr bei Herrn Prof. Dr. Leschik für die Überlassung dieser Ergebnisse und zugleich für viele Anregungen aus gemeinsamen Diskussionen. 


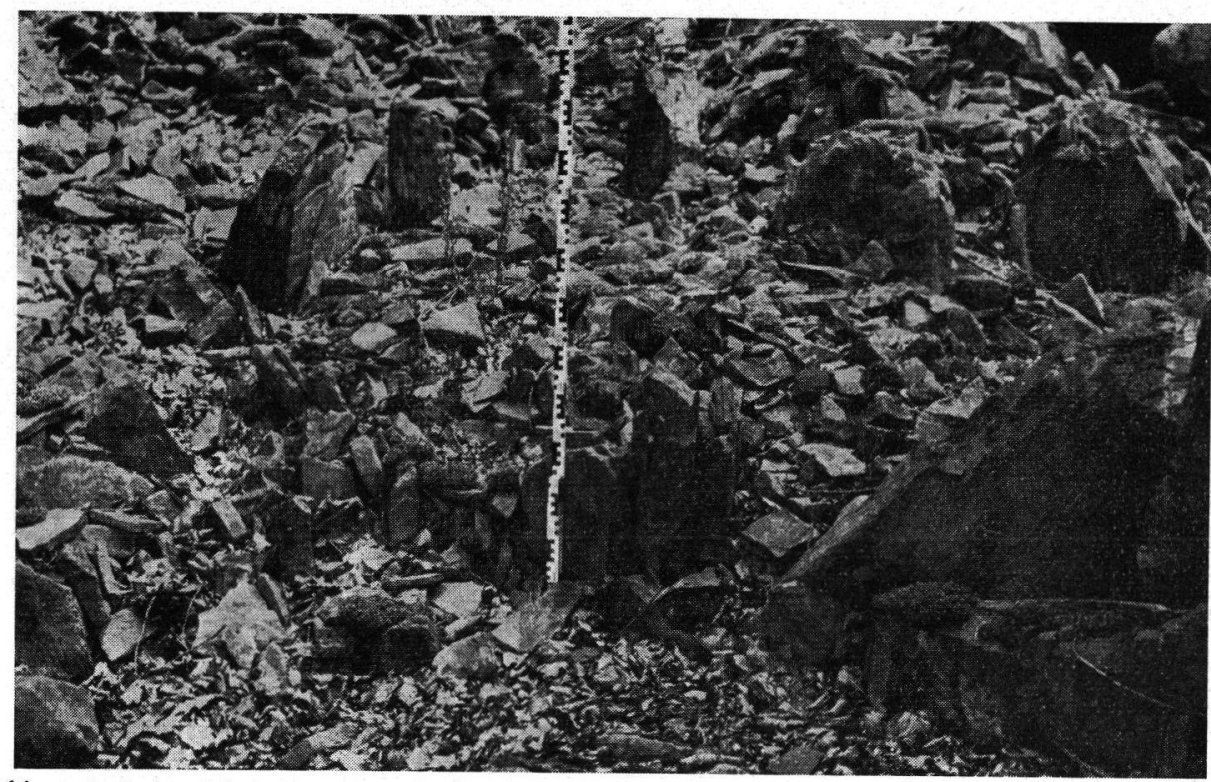

Abb. 4. Bei der Schottergewinnung im Blockmeer des Schwarzbruches stehengelassene Randsteine eines Steinringes.

der Handgewinnung von Schotter übrig geblieben war, weil die steilen Randsteine zu fest und unlösbar im Blockschutt eingekeilt waren, war auch der Anlaß der Entdeckung der Steinringe (s. Abb. 4).
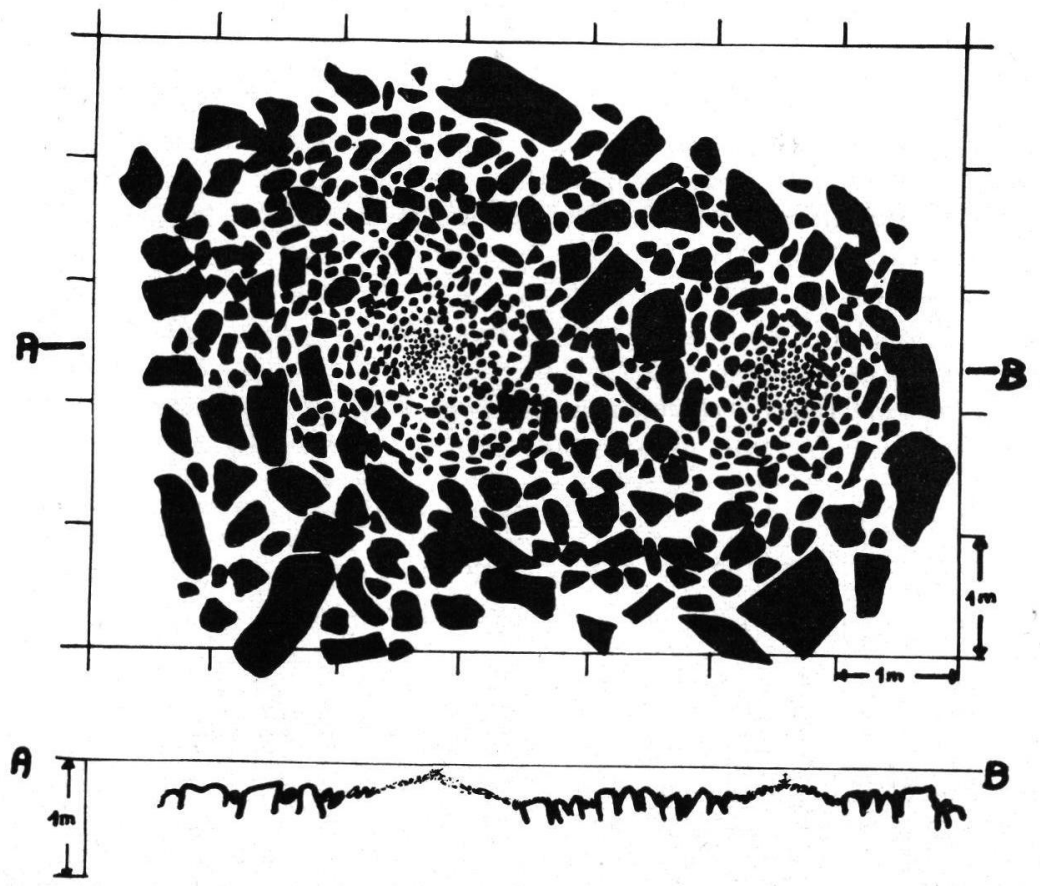

Abb. 5. Maßstäbliche Aufnahme zweier benachbarter Steinringe, Schwarzbruch bei Orscholz. Unten: Profil. 


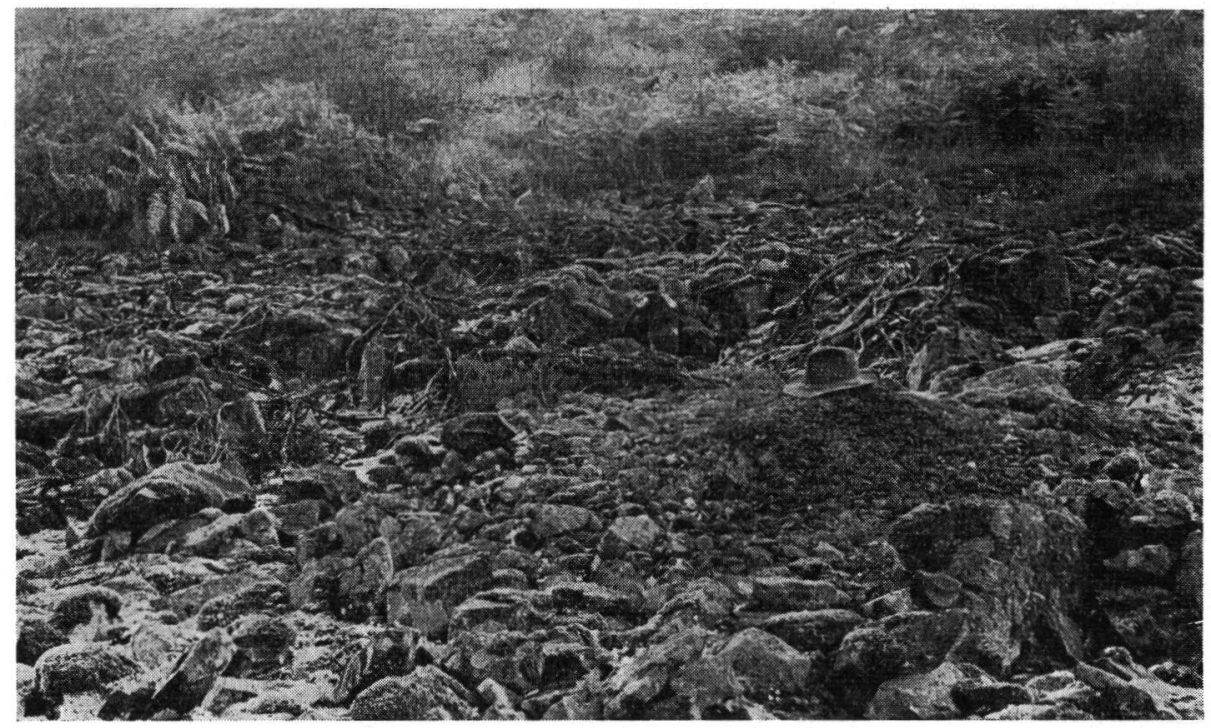

Abb. 6. Steinring. Schwarzbruch bei Orscholz.

In Abbildung 5 wird eine maßstäbliche Aufnahme zweier benachbarter Steinringe gegeben, die aus dem gleichen Vorkommen stammen.

Ein weiteres Beispiel zeigt Abb. 6.

Im ganzen konnten bislang im Blockmeer des Schwarzbruches 50 derartige Steinringe heobachtet und eingemessen werden. Die genaue Aufnahme zeigt ihre Lage vornehmlich in den flacheren Anteilen des in Abb. 3 in größerem Zusammenhang dargestellten Blockstromes. Man erkennt eine sehr benachbarte Lage der Steinringe bei einer gegenseitigen Entfernung der Ringzentren zwischen 3 und $15 \mathrm{~m}$. Da einmal durch Schotterentnahme wahrscheinlich mehrere Ringe zerstört sind, andererseits Teilgebiete des Blockstromes des Schwarzbruches dicht von Vegetation bedeckt sind, braucht die Zahl der beobachteten Steinringe nicht endgültig zu sein. Wichtig mag auch sein, daß in den oberen Anteilen des gut aufgeschlossenen Blockstromes keine Steinringe festzustellen waren.

Das Blockmeer des Schwarzbruches zeigt die Steinringe am deutlichsten und auch häufigsten, während die übrigen Blockmeervorkommen weniger ausgebildete Steinringe enthalten. $\mathrm{Zu}$ erwähnen bleiben die Steinringe in Blockströmen des Lindenhübels (bei Lebach), die aus Melaphyr bestehen und wo die kleineren Steinringe auf einer brachen Hochfläche Anlaß zur Deutung als vorgeschichtliches Gräberfeld gegeben hatten.

III.

Deutung und Bedeutung der Blockmeere und der damit verbundenen Erscheinungen

Im Vorgehenden wurde absichtlich der Wert nur auf die Darstellung von Form und Lage der Steinmeere und der damit verbundenen Erscheinungen und Strukturen gelegt und jedc Deutung vermieden, die erst jetzt gegeben werden soll. Die Blockmeere mit ihren eigentümlichen Strukturen, die in unserem Gebiet vorkommen, sind Gebilde, die unter der heutigen exogenen Bedingungen nicht entstehen oder in Bildung bleiben. Einmal fehlt der Fortgang der Gesteinszertrümmerung entlang häufiger Spaltenbildung als Urlieferant des Gesteinsschuttes, dann aber ist auch keine Fortbewegung der Hangschuttmassen und Blockmeere festzustellen. Nach vielen Merkmalen der bedeckenden Vegetation, Wald- 
bodenbildung, ja Vermoorungen sind die genannten Bildungen in der Vorzeit unter total vom heutigen Klima verschiedenen Bedingungen entstanden. Nach Analysen der Formen und Analogie mit rezenten Erscheinungen entstehen solche Bildungen nur bei tiefer Bodengefrornis und lange andauerndem Dauerfrost, also unter Bedingungen, wie sie in den heutigen periarktischen Gebieten herrschen. Sie gehören zu dem Formenschatz der periglazialen Erscheinungen, wie die Eiskeile und Brodelböden, die während des Eiszeitalters in unserem Gebiet entstanden sind, sind also Zeugen eines den heutigen periarktischen Bedingungen ähnlichen oder sogar gleichen Klimas. Das ganze Erscheinungsbild der geschilderten Formen vom durch Spaiten zertrümmerten Fels an über den Hangschutt zu den Blockmeeren und ihren Strukturen ist ohne Zerstörung der Kleinformen so gut erhalten, daß es ein getreues und lückenloses Abbild der eiszeitlichen Vorgänge in unserem Gebiet darstellt und bis in Einzelheiten der Definition der „Frostschuttzone“ (BüDEL 1950) entspricht. Die heute noch in unveränderter Form sichtbare Zerspaltung des anstehenden Hartsteins entspricht den Bildungen des Spaltenfrostes, der Mantel der Berge aus Hangschutt dem Frostschutt und die Blockmeere den Solifluktionsvorgängen in den heutigen periarktischen Gebieten. Die Steinringe z. B. des Schwarzbruches sind ebenfalls Bildungen, wie sie aus den periarktischen Gebieten z. B. aus Grönland und Spitzbergen beschrieben werden. So werden auch neuerdings aus Idaho, USA, unseren Blockmeeren und Steinringen entsprechende Bildungen der Vorzeit mit den rezenten Stein-Musterböden (patterned ground) von Thule, Grönland verglichen (MAIDE 1961).

Nach allen Merkmalen verdanken die geschilderten Bildungen der Blockmeere unseres Gebietes ihre Entstehung dem Eiszeitalter. Der erwähnte Nachweis einer Tonablagerung aus dem Riß/Würm-Interglazial unter einer Blockmeerbildung läßt darauf schließen, daß die bei uns beobachtbaren Bildungen der Würmeiszeit angehören. Dieses Alter ist auch deswegen zu unterstellen, da die stets kräftigen Denudationsvorgänge einer Eiszeit ältere Formen und besonders die Kleinformen der Steinringe zerstört hätten. Im Einzelnen ist abzuleiten, daß schon die zeitliche Aufeinanderfolge im Bildungsvorgang der Blockmeere und Steinringe auf verschiedene Kältestadien des Klimas während der letzten Eiszeit hindeuten, ähnlich wie Edelmann und Zandstra (1954) an dem Auftreten niveoäolischer Sande im Saargebiet ableiten.

Die rezenten Blockmeere und entsprechende Bildungen liegen in Gebieten mit einem Temperatur-Jahresmittel von $\mathrm{rd} .-2^{\circ} \mathrm{C}$, einem Dauerfrostboden und in einer KlimaZone, die als Frostschutt-'Tundra zu bezeichnen ist. Ähnliche Klimafaktoren und Landschaften sind für das Eiszeitalter in unserem Gebiet zu unterstellen.

Im Vorstehenden wurde ausgeführt, daß die Erscheinungen der Blockmeere und ihrer Strukturen Vorzeitformen aus dem Eiszeitalter und wahrscheinlich zu großem Teil aus der letzten Eiszeit darstellen. Andererseits zeigt die fast unveränderte Erhaltung der Großformen, aber besonders der Kleinformen, das Fehlen einer Uberprägung oder Veränderung durch jüngere Vorgänge. Hieraus kann nur abgeleitet werden, daß in unserem Gebiet die Entstehungszeit unserer Oberflächenformen im groBen wie im kleinen in das Eiszeitalter zu verlegen ist, und die Formgebung dieser Entstehungszeit an vielen Stellenganz unverändert geblieben ist, somit den Formbildungsvorgängen in der Nacheiszeit und Jetztzeit keine große Bedeutung für die Entstehung neuer Oberflächenformen zukommt, und unsere I. andschaftsform in weiten Gebieten die konservierte eiszeitliche Landoberfläche mit sämtlichen morphologischen Einzelheiten darstellt.

Die Abtragungsprodukte des Eiszeitalters sind in dem Frostschuttmantel der Berge und den Blockmeeren nur teilweise erhalten und zu einem Großteil in die Täler transportiert, wo sie in den Schottern der Talterrassen erhalten sind. Wenn man aber bedenkt, daß der noch erhaltene Verwitterungsschutt der Berge mit seinen Mächtigkeiten doch 
Beträge annimmt, die mitunter eine Blockschuttmasse von 100000 cbm pro Hektar übersteigen, wird erst recht die hohe Abtragungsleistung des Eiszeitalters in unserem Gebiet gut augenfällig. Im Gegensatz zu den Tälern, von denen wir ebenfalls wissen, daß sie Entstehung und Form den eiszeitlichen Vorgängen verdanken, und in denen eine mehr linienhafte Erosion wirksam war, zeigen die Erscheinungen der talferneren Bereiche die große flächenhafte Abtragungswirkung des Eiszeitklimas auf. Die Abtragungsleistung des Eiszeitklimas in unseren Periglazialgebieten darf mit der in den heutigen periarktischen Gebieten ohne weiteres verglichen werden, von der BünEL (1950) darlegt, daß sie in Bezug auf die quantitative Abtragungsleistung von keiner anderen Klimazone der Erde übertroffen wird.

Die Darlegungen wollen nicht so verstanden sein, als ob in unserem Gebiet keine Vorgänge einer neuen Formgestaltung unter dem Einfluß des jetzigen Klimas mehr wirksam wären. Die Tätigkeit des Wassers besonders ist einer der heute auf die Oberfläche wirksamen Faktoren. Auch Frostbildungen treten bei uns heute noch auf (SELzER 1959), doch ist die Wirkung der in der kurzen Nacheiszeit bestehenden Kräfte nicht mit denen des Eiszeitalters zu vergleichen.

\section{IV.}

Vorzeitliche Fliehburgen und Blockmeere

Die Untersuchung der einheimischen Blockmeervorkommen ergab einen weiteren mittelbaren Zusammenhang mit der einheimischen Geschichte. Die Vorzeitburgen unseres Gebietes (STEINER 1932) sind entweder von Erddämmen umgeben, oder aus Steinwällen errichtet, wobei den aus Steinwällen errichteten Burgen nach Größe und Zahl die größte Bedeutung zukommt. Wenn man bedenkt, daß die Gesteinsmasse der Ringwälle des Hunnenrings bei Otzenhausen mehrere $100000 \mathrm{cbm}$ unfaßt, und daß nur Gesteinsbrocken von über $1 \mathrm{dm}$ Größe benutzt worden sind, ist es verwunderlich, daß noch nie die Frage nach der Herkunft dieser Steine gestellt worden ist. Das Steinmaterial der Ringwälle unseres Gebietes ist in den meisten Fällen Hartstein aus Taunusquarzit (Hunnenring) oder Melaphyr, auf jeden Fall schwerst bearbeitbare Gesteine. Es nimmt somit kein Wunder, daß entsprechende Steinbrüche ganz fehlen.

Es ist nun eigentümlich, daß sich die Vorzeitburgen aus Steinwällen unseres Gebietes (auch des ganzen Hunsrücks) nur an den Stellen der Vorkommen eiszeitlicher Blockmeere finden, und daß danach der vorzeitliche Mensch ausgesprochen solche Stellen zu Fliehburgen ausgewählt hat, an denen das entsprechende Steinmaterial in entsprechender Form zu finden war. So zeigt sich am Hunnenring, Mommerich und Donnersberg, daß die Fliehburgen innerhalb von Blockmeervorkommen gelegen sind, daß z. B. die Steine eine gewisse Abrundung zeigen, und daß nur ein Bruchteil des natürlich vorhandenen Materials benutzt worden ist. Selbst die Anordnung der Steinwälle besonders in Hanglage, läßt vermuten, daß vorhandene Schutt-Terrassen, die ja meist hangparallel verlaufen, vom Vorzeitmenschen in situ nur umgebaut worden sind.

Für die Hangwälle des Hunnenringes trifft diese Eigenart der Ausbildung von SchuttTerrassen ganz ausgesprochen zu.

\section{$\mathrm{Schriftenverzeichnis}$}

Büdel, J.: Das System der klimatischen Morphologie. - Deutscher Geographentag München 1948, Landshut 1950.

Edelmann, C. H. \& Zandstra, K. J.: Niveo-äolische Sande im Saargebiet. - Proc. kon. nederl. Akademie van Wetensch., Ser. B, 59, N. 3, Amsterdam 1956.

FisCHer, F.: Beiträge zur Morphologie des Flußsystems der Saar. - Ann. Univ. Saravensis 5, F. 2. 1956. 
Kessler, P.: Das eiszeitliche Klima und seine Wirkungen im nicht vereisten Gebiet. - Stuttgart 1925. Kessler, P.: Frostspalten bei Saarbrücken. - Z. deutsch. geol. Ges. 79, 1927.

MALDE, H. E.: Patterned ground of possible solifluction origin at law altitude in the western Snake river plain, Idaho. - Geol. Surv. Res. 1961, Short papers in the Geol. and Hydrol. Sciences. Washington 1961.

Mathias, K.: Morphologie des Saartales zwischen Saarbrücken und der Saarmündung. - Verh. naturhist. Ver. Rheinl. u. Westf. 93, 1936.

Mertis, C.: Glaziale Erscheinungen im Haardtgebirge. - Globus 1886.

Poser, H.: Auftautiefe und Frostzerrung im Boden Mitteleuropas während der Würm-Eiszeit. Die Naturwissenschaften 34, Berlin 1947.

Sснотт, C.: Die Blockmeere in den deutschen Mittelgebirgen. - Forsch. z. deutschen Landes- u. Volkskunde 29, 1931.

Selzer, G.: Erdkegel als heutige Frostbodenbildungen im Saarland. - Eiszeitalter und Gegenwart 10, 1959.

Steiner, P.: Die Vorzeitburgen des Hochwaldes. - Trier 1932.

WoldSTEDT, O.: Das Eiszeitalter, Bd. II. - Stuttgart 1958.

ZANDSTRA, K. J.: Die jungquartäre morphologische Entwicklung des Saartales. - Erdkunde 8, 1954.

Manuskr. eingeg. 18. 3. 1963.

Anschrift des Autors: Dr. Georg Selzer, Ensheim/Saar, Geol. Landesamt. 